INPLASY

PROTOCOL

To cite: Wang et al. Clinical Efficacy of Rhythm vs Rate Control in Patients with Atrial Fibrillation(AF) Based on 16 Trials: A Meta-Analysis. Inplasy protocol 202110093. doi: 10.37766/inplasy2021.1.0093

Received: 23 January 2021

Published: 24 January 2021

Corresponding author: Zhaobo Wang

wangzhaobo2020@sina.com

Author Affiliation:

Graduate School of

Heilongjiang University Of

Chinese Medicine

Support: The NNSF of

China(81473517).

Review Stage at time of this submission: Preliminary searches.

Conflicts of interest:

None.

\section{Clinical Efficacy of Rhythm vs Rate Control in Patients with Atrial Fibrillation(AF) Based on 16 Trials: A Meta-Analysis}

Wang, Z1; Li, Y2; Liu, Y3; Yang, T4; Yu, Y5; Wang, J6; Chi, Y7.

Review question / Objective: P:Patients with Atrial Fibrillation(AF); I:Rhythm Control; C:Rate Control; O: 1.Exercise Load Test, Distance of $6 \mathrm{~min}$ Walk Tests, Hospitalization, QOL, Echocardiography, Mortality etc.; S:RCT.

Condition being studied: Atrial Fibrillation(AF). At present, the issue is controversial and there is no clear recommendation in the Guidelines. Existing meta analysis didn't include sufficient trials and outcome.

Information sources: Electronic database(Web of Science, Embase, PMC)

INPLASY registration number: This protocol was registered with the International Platform of Registered Systematic Review and Meta-Analysis Protocols (INPLASY) on 24 January 2021 and was last updated on 24 January 2021 (registration number INPLASY202110093).

\section{INTRODUCTION}

Review question / Objective: P:Patients with Atrial Fibrillation(AF); I:Rhythm Control; C:Rate Control; O: 1. Exercise Load Test, Distance of $6 \mathrm{~min}$ Walk Tests,
Hospitalization, QOL, Echocardiography, Mortality etc.; S:RCT.

Condition being studied: Atrial Fibrillation(AF). At present, the issue is controversial and there is no clear recommendation in the Guidelines. Existing 
meta analysis didn't include sufficient trials and outcome.

\section{METHODS}

Participant or population: Patients with Atrial Fibrillation(AF) $=10723$.

Intervention: Rhythm Control.

\section{Comparator: Rate Control.}

Study designs to be included: RCT.

Eligibility criteria: A randomized controlled trials of atrial fibrillation. The results of this study were compared between the Rhythm control and Rate Control group.Data is complete and extractable.

Information sources: Electronic database(Web of Science, Embase, PMC).

Main outcome(s): Exercise Load Test, Distance of $6 \mathrm{~min}$ Walk Tests, QOL, Mortality.

\section{Additional outcome(s): Embolic Events,} Echocardiography, Hospitalization.

Data management: The Meta analysis of counting and continuous data is carried out by Stata15.0 software. The counting data are expressed as $\mathrm{OR}$ and $95 \% \mathrm{Cl}$, and the continuous data are expressed as SMD and $95 \% \mathrm{Cl}$.

Quality assessment / Risk of bias analysis: The Jadad score, which indicates the quality of studies based on their description of randomization, blinding, and dropouts (withdrawals), was used to assess the methodological quality of trials. The quality scale ranges from 0 to 5 points, with a score of $\leq 2$ indicating a low quality report, and a score of $\geq 3$ indicating a high quality report.

Strategy of data synthesis: In the count class data, we studied the proportion of positive events.Continuous data need to be presented in the form of Difference of "Mean \pm SD"( before and after treatment).

Subgroup analysis: By type of disease and complications.

Sensitivity analysis: We used Stata15.0 software for sensitivity analysis. When the effect exceeds $95 \% \mathrm{Cl}$ upper and lower limits, or $95 \% \mathrm{Cl}$ upper and lower limits exceed the mean, this study is the main factor of heterogeneity.

Language: English.

Country(ies) involved: China.

Keywords: Atrial Fibrillation, Rhythm Control, Rate Control, Meta-analysis.

Contributions of each author:

Author 1 - Zhaobo Wang.

Email: wangzhaobo2020@sina.com

Author 2 - Yingying Li.

Email: lyy18801095040@163.com

Author 3 - Ying Liu.

Email: LY122608@163.com

Author 4 - Tao Yang.

Email: 630836504@qq.com

Author 5 - Yixuan Yu.

Email: yuyixuankx@163.com

Author 6 - Junzhi Wang.

Email: drvolodia@163.com

Author 7 - Yuan Chi.

Email: 1972337065@qq.com 